\title{
Proposed physical explanation for the electron spin and related antisymmetry
}

\author{
A. M. Cetto, L. de la Peña and A. Valdés-Hernández \\ Instituto de Física, Universidad Nacional Autónoma de México
}

January 3, 2018

\begin{abstract}
We offer a possible physical explanation for the origin of the electron spin and the related antisymmetry of the wave function for a two-electron system, in the framework of nonrelativistic quantum mechanics as provided by linear stochastic electrodynamics. A consideration of the separate coupling of the electron to circularly polarized modes of the random electromagnetic vacuum field, allows to disclose the spin angular momentum and the associated magnetic moment with a $g$-factor 2 , and to establish the connection with the usual operator formalism. The spin operator turns out to be the generator of internal rotations, in the corresponding coordinate representation. In a bipartite system, the distinction between exchange of particle coordinates (which include the internal rotation angle) and exchange of states becomes crucial. Following the analysis of the respective symmetry properties, the electrons are shown to couple in antiphase to the same vacuum field modes. This finding, encoded in the antisymmetry of the wave function, provides a physical rationale for the Pauli principle. The extension of our results to a multipartite system is briefly discussed.

Keywords: Pauli principle; electron spin; spin-symmetry connection; stochastic electrodynamics; bipartite entanglement; zero-point radiation field.
\end{abstract}

\section{Introduction}

Can one speak of the origin of the electron spin? Does it make sense to look for a physical agent behind the exclusion principle? These two most prominent features of the electron are customarily taken in nonrelativistic quantum mechanics as empirical facts. Nevertheless, the concept of spin as a derived quality rather than an innate one has given rise over the years to a diversity of suggestive proposals, as is illustrated by our short but multifarious selection of relevant work [1]- 12]. On the other hand, despite the various proofs existing in the literature, the physical gears behind the spin-statistics connection are still unclear (10]-[1] and references therein). Here we address this state of affairs in 
an integrated approach using the tools of stochastic electrodynamics (SED), the theory developed to explain the quantum behavior of matter as a result of its interaction with the fluctuating radiation vacuum or zero-point field (ZPF) [13], taken as a real field. In other words, the purpose of this paper is to reveal the physics behind the spin and its symmetry properties, in contrast to relativistic quantum mechanics, where the electron spin appears as a natural element of the formalism.

It should be noted that the electron spin has received some, though rather limited attention in SED. In particular, by using a harmonic-oscillator model for the particle and separating the ZPF into components of circular polarization, de la Peña and Jáuregui [5] obtained the spin angular momentum and the associated magnetic moment as acquired properties, the former within a numerical factor of order 1. Sachidanandam [6] arrived at similar results for an electron in a uniform magnetic field. More recently, Muralidhar [7] derived the electron spin by taking the zero-point energy of the (free) electron as an energy of rotation within the region of space surrounding the particle. These various SED-inspired calculations are quite suggestive, in that they all lead to a result of order $\hbar^{2}$ for the mean square value of the spin and of order $\hbar$ for the spin projections, exhibiting the ZPF as the source of a kind of (nonrelativistic) zitterbewegung. This confirms the recurrent proposal of a close relationship between spin and zitterbewegung, which started with Schrödinger and extends to our days, as is exemplified in the cited literature[1]-[13].

Here we use the tools provided by linear stochastic electrodynamics (LSED) to tackle the issue of the spin-statistics connection for a two-electron system, which leads us to propose a specific physical mechanism for the Pauli exclusion principle, not examined in previous work. We start by recalling that according to LSED, the spin appears as an intrinsic angular momentum of size $\hbar / 2$, with an associated magnetic moment with $g$-factor $g_{S}=2[9]$. This paves the way for the introduction of the corresponding spin-operator formalism and for establishing contact with its usual (nonrelativistic) quantum treatment. We then recall that two identical particles forming part of the same system couple to the same mode of the vacuum field; this is the mechanism behind the entanglement of their state vector, as indicated previously ([13]-[14]). The present work goes further with respect to Refs. 9], 14, [15, in that here we take advantage of the fact that the LSED expressions still contain explicitly the relevant field variables describing the spin orientation, and show that a proper consideration of the rotation angle associated with the spin angular momentum, combined with a careful analysis of the exchange properties of the (entangled) state vector, leads to the antisymmetry of the latter. This result reveals an antiphase coupling of the two electrons to the common field modes. Since no more than two particles can couple in antiphase to the same mode, we disclose here the origin of the Pauli principle. The analysis can be extended to a multi-electron system, as is briefly discussed in the final part of the paper.

For a systematic and comprehensive account of SED in its present form and in particular for a detailed explanation of the emergence of entangled states, we refer the reader to Refs. [13] and [14, respectively. Ref. 9] contains previous 
work on the emergence of spin in LSED. Additionally, some results presented in this paper draw from previous work on the subject, specifically Ref. [15].

\section{Intrinsic angular momentum of the vacuum field}

Let us start by recalling from the theory of electric dipole transitions in atoms that in a single transition the absorbed or emitted radiation carries one unit of angular momentum, involving an interaction of the electron with (photonic or external) radiation field modes of circular polarization [16. Since in SED we work normally in the electric dipole approximation, we shall assume that the coupling of electrons to the ZPF also involves circularly polarized field modes. Accordingly, we expand the ZPF vector potential in terms of plane modes of frequency $\omega_{k}=c|\boldsymbol{k}|$, wave vector $\boldsymbol{k}$, and circular polarization $\gamma$ (see e. g. Ref. [17, section 10.6),

$$
\boldsymbol{A}(\boldsymbol{r}, t)=\frac{1}{2} \sqrt{\frac{\hbar}{V}} \sum_{\boldsymbol{k}, \gamma} \frac{1}{\sqrt{\omega}}\left[-i \hat{\boldsymbol{\epsilon}}_{\boldsymbol{k}}^{\gamma}(\varphi) a(\boldsymbol{k}, \gamma) e^{i(\boldsymbol{k} \cdot \boldsymbol{r}-\omega t)}+\text { c.c. }\right],
$$

with $V$ the volume of integration. The permittivity $\epsilon_{0}$ of free space is taken equal to 1 , and $\omega=\omega_{k}$. The intensity of the field is proportional to Planck's constant, which fixes the energy per normal mode at $\hbar \omega / 2$, as corresponds to the zero-point term (see below).

Let $\hat{\boldsymbol{e}}_{i}, i=1,2,3$, be a right-handed triadic of Cartesian unit vectors, with $\hat{\boldsymbol{e}}_{3}$ pointing in the direction of $\boldsymbol{k}$; the right and left circular polarization vectors are then given by $\hat{\epsilon}_{\boldsymbol{k}}^{+}(\varphi)=\frac{1}{\sqrt{2}}\left(\hat{\boldsymbol{e}}_{1}+i \hat{\boldsymbol{e}}_{2}\right) e^{i \varphi}, \hat{\epsilon}_{\boldsymbol{k}}^{-}(\varphi)=\frac{i}{\sqrt{2}}\left(\hat{\boldsymbol{e}}_{1}-i \hat{\boldsymbol{e}}_{2}\right) e^{-i \varphi}$, where $\varphi$ is the angle of rotation around the $\hat{\boldsymbol{e}}_{3}$-axis. Further, the mode amplitudes $a(\boldsymbol{k}, \gamma)=e^{i \zeta(\boldsymbol{k}, \gamma)}$ vary at random from realization to realization of the field; this is where stochasticity comes in. For a maximally incoherent field such as the free ZPF, the phases pertaining to different modes $(\boldsymbol{k}, \gamma)$ are statistically independent. It is convenient to absorb the $\varphi$-factors appearing in the polarization vectors $\hat{\boldsymbol{\epsilon}}_{\boldsymbol{k}}^{\gamma}(\varphi)$ into the mode amplitudes, so that the latter become

$$
a(\boldsymbol{k}, \gamma, \varphi)=e^{i \zeta(\boldsymbol{k}, \gamma)} e^{i \gamma \varphi(\boldsymbol{k}, \gamma)}, \quad \gamma= \pm 1
$$

and the polarization vectors reduce to $\hat{\boldsymbol{\epsilon}}_{\boldsymbol{k}}^{+}=\frac{1}{\sqrt{2}}\left(\hat{\boldsymbol{e}}_{1}+i \hat{\boldsymbol{e}}_{2}\right), \hat{\boldsymbol{\epsilon}}_{\boldsymbol{k}}^{-}=\frac{i}{\sqrt{2}}\left(\hat{\boldsymbol{e}}_{1}-i \hat{\boldsymbol{e}}_{2}\right)$. Equation (11) takes then the form

$$
\boldsymbol{A}(\boldsymbol{r}, t)=\frac{1}{2} \sqrt{\frac{\hbar}{V}} \sum_{\boldsymbol{k}, \gamma} \frac{1}{\sqrt{\omega}}\left[-i \hat{\boldsymbol{\epsilon}}_{\boldsymbol{k}}^{\gamma} a(\boldsymbol{k}, \gamma, \varphi) e^{i(\boldsymbol{k} \cdot \boldsymbol{r}-\omega t)}+\text { c.c. }\right] .
$$

Integrating over the entire volume, with the help of Eq. (2), one readily obtains for every mode contained in (3) a fixed (nonrandom) energy $H_{\boldsymbol{k}}^{\gamma}=$ 
$\hbar \omega / 2=\hbar \omega_{\boldsymbol{k}} / 2$, a nonrandom linear momentum $\boldsymbol{P}_{\boldsymbol{k}}^{\gamma}=\hbar \omega \hat{\mathbf{k}} / 2 c$, and a fixed intrinsic angular momentum along the direction of propagation $\boldsymbol{J}_{k}^{\gamma}$ of value

$$
\boldsymbol{J}_{\boldsymbol{k}}^{\gamma}=\int_{V}(\boldsymbol{E} \times \boldsymbol{A})_{\boldsymbol{k}}^{\gamma} d^{3} r=\gamma \frac{\hbar}{2} \hat{\mathbf{k}}, \quad \gamma= \pm 1 .
$$

These values coincide with the results reported in the literature 17]. Since there are as many modes in the $\boldsymbol{k}$ direction as in the $-\boldsymbol{k}$ direction, the total linear momentum vanishes for every $\omega$. Further, for the free field the contributions of the two polarizations compensate each other for every $\boldsymbol{k}$, so that the total intrinsic angular momentum vanishes as well. This may explain why, in SED as well as in QED, these terms are normally omitted. Yet every individual field mode $(\boldsymbol{k}, \gamma)$ does have an intrinsic angular momentum of value $\pm \hbar / 2$ along the direction of propagation, according to Eq. (44). This decomposition of the radiation field into orbital and intrinsic (spinorial) components is legitimated by the fact that for a free electromagnetic field the intrinsic angular momentum is a constant of the motion; a detailed discussion of this matter can be seen in Ref. [17, Section 10.6.3.

\section{Intrinsic angular momentum of the particle}

For an analysis of the effect of the vacuum field on the angular momentum of the electron, the approach provided by LSED is particularly convenient. As shown elsewhere (Ref. [13] and references therein) the theory furnishes a description of the stationary states of the mechanical system once it has reached the quantum regime - i.e., when the system has acquired ergodic properties and detailed energy balance has been attained between particle and vacuum field. Additional effects of the radiation terms are then negligible and may be omitted in the radiationless approximation. The ensuing description is, formally, entirely equivalent to the Heisenberg quantum description; yet it still contains relevant information about those field modes that play a central role in sustaining the stationary states. This information also plays a key role in the analysis that follows.

Let us consider a charged, pointlike particle subject to an external conservative force, typically an atomic electron. According to LSED, a generic dynamical variable $G(t)$ pertaining to the particle in a stationary state $\alpha$ has the form

$$
G_{\alpha}(t)=\tilde{G}_{\alpha \alpha}+\sum_{\beta \neq \alpha} \tilde{G}_{\alpha \beta} a_{\alpha \beta} e^{i \omega_{\alpha \beta} t},
$$

where the index $\beta \neq \alpha$ represents any other stationary state; the set $\{\alpha \beta\}$ depends on the specific problem. The $\tilde{G}_{\alpha \beta}$ turn out to be the matrix elements (in the energy representation) of the respective quantum operator $\hat{G}$, so that $\tilde{G}_{\alpha \alpha}$ is the expectation value of $G$ in state $\alpha$. The field amplitudes $a_{\alpha \beta}$ pertain to those modes of the field (of frequency $\omega_{\alpha \beta}$ ) to which the particle responds resonantly, and are given by (cf. Eq. (2))

$$
a_{\alpha \beta}(\varphi)=e^{i \zeta_{\alpha \beta}} e^{i \gamma_{\alpha \beta} \varphi}, \quad \gamma_{\alpha \beta}= \pm 1 .
$$


As manifested by the dependence of $G_{\alpha}$ on the $a_{\alpha \beta}$, the particle variables are driven linearly, so to say, by such field modes (under stationarity neither $\tilde{G}_{\alpha \beta}$ nor $\omega_{\alpha \beta}$ depend on the stochastic amplitudes $a_{\alpha \beta}$ ). A notable feature of the quantum regime is that when ergodicity is imposed, the phases of the $a_{\alpha \beta}$ become correlated in such a way that the chain rule $a_{\alpha \beta^{\prime}} a_{\beta^{\prime} \beta}=a_{\alpha \beta}$ holds (no summation over repeated indices) [13, with $\beta^{\prime}$ any stationary state, which implies $\zeta_{\alpha \beta}=\zeta_{\alpha}-\zeta_{\beta}, \omega_{\alpha \beta}=\omega_{\alpha}-\omega_{\beta}$, and $\gamma_{\alpha \beta}=\gamma_{\alpha}-\gamma_{\beta}$. Physically this is a result of the effect of the radiating particle on the field under stationary conditions; mathematically, this guarantees that the product of two (or more) dynamical variables can also be written as a linear expansion of the form (5). Further, the relation $\omega_{\alpha}=\mathcal{E}_{\alpha} / \hbar$ is shown to hold, with $\mathcal{E}_{\alpha}$ the energy associated with state $\alpha$, meaning that the resonance frequencies are just the transition frequencies as given by Bohr's rule [13].

Equation (5) applies in particular to the components of $\boldsymbol{x}(t)$ and $\boldsymbol{p}(t)=$ $m \dot{\boldsymbol{x}}(t)$ in state $\alpha$. The average value of the angular momentum component $L_{z}=x p_{y}-y p_{x}$ becomes thus

$$
\left\langle L_{z}\right\rangle_{\alpha}=\left\langle\alpha\left|\hat{L}_{z}\right| \alpha\right\rangle=i m \sum_{\beta} \omega_{\beta \alpha}\left(\tilde{x}_{\alpha \beta} \tilde{y}_{\beta \alpha}-\tilde{y}_{\alpha \beta} \tilde{x}_{\beta \alpha}\right),
$$

with $\tilde{x}_{\alpha \beta}=\langle\alpha|\hat{x}| \beta\rangle, \tilde{y}_{\alpha \beta}=\langle\alpha|\hat{y}| \beta\rangle$, and $|\alpha\rangle$ a vector in $\mathcal{H}$, the corresponding Hilbert space of states.

Since the electron is driven by the (electric component of the) circularly polarized ZPF modes, we shall consider separately the two circular polarizations of the field modes contributing to $\left\langle L_{z}\right\rangle_{\alpha}$, which are those that propagate along the $z$ axis. It is therefore convenient to use cylindrical variables for the particle: $x^{+}=\boldsymbol{x} \cdot \hat{\boldsymbol{\epsilon}}^{+}=\frac{1}{\sqrt{2}}(x+i y), x^{-}=\boldsymbol{x} \cdot \hat{\boldsymbol{\epsilon}}^{-}=\frac{1}{\sqrt{2}}(i x+y)$, and $x^{k}=z$. In terms of these, (7) rewrites as

$$
\left\langle L_{z}\right\rangle_{\alpha}=m \sum_{k} \omega_{\beta \alpha}\left(\left|\tilde{x}_{\alpha \beta}^{+}\right|^{2}-\left|\tilde{x}_{\alpha \beta}^{-}\right|^{2}\right) .
$$

To calculate the separate terms in (8) we resort to the commutator $[\hat{x}, \hat{p}]=$ $i \hbar$, which in LSED is shown to be a further consequence of the particle-field interaction in the quantum regime. 15] In cylindrical variables it takes the form

$$
\hbar=m \sum_{\beta} \omega_{\beta \alpha}\left(\left|\tilde{x}_{\alpha \beta}^{+}\right|^{2}+\left|\tilde{x}_{\alpha \beta}^{-}\right|^{2}\right) .
$$

By combining with Eq. (8) we get

$$
\left\langle L_{z}\right\rangle_{\alpha}=\left\langle M_{z}\right\rangle_{\alpha}^{+}+\left\langle M_{z}\right\rangle_{\alpha}^{-},
$$

with

$$
\left\langle M_{z}\right\rangle_{\alpha}^{+}=\frac{1}{2}\left\langle L_{z}\right\rangle_{\alpha}+\frac{1}{2} \hbar, \quad\left\langle M_{z}\right\rangle_{\alpha}^{-}=\frac{1}{2}\left\langle L_{z}\right\rangle_{\alpha}-\frac{1}{2} \hbar .
$$

Notice that even if (and when) $\left\langle L_{z}\right\rangle_{\alpha}$ is zero, the separate contributions to $\left\langle M_{z}\right\rangle_{\alpha}^{+}$and $\left\langle M_{z}\right\rangle_{\alpha}^{-}$do not vanish. Each one contains one-half of the orbital 
angular momentum along the $z$ direction plus an intrinsic angular momentum component $\pm \hbar / 2$ associated with either one or the other polarization; this intrinsic term we identify with the spin component $S_{z}$. By summing over the polarizations, the spin terms cancel each other and we are left with the orbital term only. So even though its contributions are concealed by the summation, the spin emerges as manifestation of the coupling of the particle to the separate polarized field modes of the ZPF.

To express $\left\langle M_{z}\right\rangle_{\alpha}^{ \pm}$as the average of an operator $\hat{M}_{z}$, we note that $\left\langle L_{z}\right\rangle_{\alpha}$ does not depend on the spin state and the latter does not depend on the atomic state. We therefore decompose $\mathcal{H}$ as $\mathcal{H}=\mathcal{H}_{0} \otimes \mathcal{H}_{S}$, with $\mathcal{H}_{0}$ the non-spin Hilbert space, spanned by the orbital state vectors, denoted henceforth by $\left|\alpha_{0}\right\rangle$, and $\mathcal{H}_{S}$ a bidimensional space spanned by the orthonormal vectors $\{|\sigma\rangle\}=(|+\rangle,|-\rangle)$ representing the eigenstates of a spin operator which we call $\hat{\boldsymbol{S}}$. In terms of $|\alpha\rangle=\left|\alpha_{0}, \sigma\right\rangle=\left|\alpha_{0}\right\rangle \otimes|\sigma\rangle$, Eqs. (11) become

$$
\left\langle\alpha\left|\hat{M}_{z}\right| \alpha\right\rangle=\frac{1}{2}\left\langle\alpha_{0}\left|\hat{L}_{z}\right| \alpha_{0}\right\rangle+\left\langle\sigma\left|\hat{S}_{z}\right| \sigma\right\rangle,
$$

with $\sigma= \pm 1$. In terms of the Pauli matrix $\hat{\sigma}_{z}$ we have $\hat{S}_{z}=\hbar \hat{\sigma}_{z} / 2$, and (12) becomes

$$
\langle\alpha|\hat{\boldsymbol{M}} \cdot \hat{\boldsymbol{z}}| \alpha\rangle=\left\langle\alpha_{0} \sigma\left|\left(\frac{1}{2} \hat{\boldsymbol{L}}+\hat{\boldsymbol{S}}\right) \cdot \hat{\boldsymbol{z}}\right| \alpha_{0} \sigma\right\rangle,
$$

where

$$
\hat{\boldsymbol{S}}=\frac{1}{2} \hbar \hat{\boldsymbol{\sigma}} .
$$

The independence of $\left\langle\hat{L}_{z}\right\rangle$ from $\sigma$ and of $\left\langle\hat{S}_{z}\right\rangle$ from $\alpha_{0}$, indicates that under the present conditions, the fluctuations associated with the spin (a non-relativistic zitterbewegung, taken as internal) are not correlated with those that characterize the mean instantaneous kinematics of the particle. This is a characteristic nonrelativistic independence. (The spaces of the two angular momenta may of course become connected by the presence of magnetic forces.) By internal we are referring to the jiggling around the local mean position of the particle; when a translational motion is superimposed, this corresponds to a kind of helicoidal motion.

Along with energy and momentum, a given mode of the ZPF is thus seen to also transfer a minimum angular momentum to the particle, equal to its mean spin value $\hbar / 2$, independent of the binding force. It should be stressed that this ineluctable angular momentum does not refer to a spinning motion of the (pointlike!) particle, but rather to a rotation around its instantaneous position along its (comparatively smooth) trajectory 11 This additional motion endorses the notion frequently encountered (already in Schrödinger, Ref. 1]) that the spin has its origin just in the zitterbewegung. At the same time it explains why the spin cannot be associated with the instantaneous mean local coordinates of the particle, since it represents a motion around the latter. Further, it elucidates the reason for the presence of spin in Dirac's equation, since this (relativistic)

\footnotetext{
${ }^{1}$ In Ref. 9], this rotational motion is shown to be generated by the torque due to the electric component of the ZPF; see Eq. (41) and the related discussion in that paper.
} 
theory predicts the zitterbewegung, a phenomenon totally ignored by contrast in the Schrödinger theory, where instead it needs to be introduced by hand.

With these elements we can readily calculate the $g_{S}$-factor associated with the electron spin — which in the nonrelativistic case is also normally introduced by hand. For this purpose consider an atomic electron acted on by a static uniform magnetic field $\boldsymbol{B}=B \hat{\boldsymbol{z}}$ in addition to the binding Coulomb force. The additional contribution to the Hamiltonian is $\hat{H}=-\hat{\boldsymbol{\mu}} \cdot \boldsymbol{B}$, with $\hat{\boldsymbol{\mu}}=$ $-\left(g_{L} \mu_{0} \hat{\boldsymbol{L}}\right) / \hbar, \mu_{0}=|e| \hbar /(2 m c)$ the Bohr magneton $(e=-|e|)$, and $g_{L}=1$. The corresponding mean energy $\mathcal{E}=\mu_{0} B\left\langle\hat{L}_{z}\right\rangle / \hbar$ can be separated using Eqs. (10) and (11) into $\mathcal{E}^{ \pm}=\mu_{0} B\left(\left\langle\hat{L}_{z}\right\rangle+2\left\langle\hat{S}_{z}\right\rangle^{ \pm}\right) / 2 \hbar$. The partial Hamiltonians describing the magnetic interaction of the electron with right and left modes are therefore $\hat{H}_{L S}^{+}=\hat{H}_{L S}^{-}=\mu_{0} B\left(\hat{L}_{z}+2 \hat{S}_{z}\right) / 2 \hbar$, and the full Hamiltonian reads

$$
\hat{H}_{L S}=\hat{H}_{L S}^{+}+\hat{H}_{L S}^{-}=\frac{\mu_{0}}{\hbar} B\left(\hat{L}_{z}+2 \hat{S}_{z}\right),
$$

with the correct $g_{S}$-factor of 2 for the spin magnetic moment. It is clear from this derivation that the value of $g_{S}$ is linked with the two polarizations of the ZPF. 5] By writing Eq. (15) as $\hat{H}_{L S}=-\hat{\boldsymbol{\mu}} \cdot \boldsymbol{B}$, the operator $\hat{\boldsymbol{M}}$ turns out to be proportional to the total magnetic moment operator $\hat{\boldsymbol{\mu}}$ of the atomic electron,

$$
\hat{\boldsymbol{\mu}}=-\frac{\mu_{0}}{\hbar}(\hat{\boldsymbol{L}}+2 \hat{\boldsymbol{S}})=-\frac{2 \mu_{0}}{\hbar} \hat{\boldsymbol{M}} \text {. }
$$

Now we reformulate the state vectors $|\alpha\rangle=\left|\alpha_{0}, \sigma\right\rangle=\left|\alpha_{0}\right\rangle \otimes|\sigma\rangle$ considering the internal rotation angle $\varphi$ introduced in section 2. As Eq. (5) indicates, $G_{\alpha}$ depends on $\varphi$ through the amplitudes (6), with $\gamma_{\alpha \beta}=\gamma_{\alpha}-\gamma_{\beta}= \pm 1$, as explained after Eq. (6). We profit from the structure of Eq. (5) to transfer the dependence on $\varphi$ to the state vectors $|\alpha\rangle$, and define

$$
|\alpha(\varphi)\rangle=e^{i \gamma_{\alpha} \varphi}\left|\alpha_{0}, \sigma\right\rangle,
$$

meaning that the angle $\varphi$ is now associated with the particle.

To determine the set of values $\left\{\gamma_{\alpha}\right\}$, let us assume that there exist (at least) three different possible values, say $\gamma_{\alpha}, \gamma_{\beta}$, and $\gamma_{\delta}$. Then $\gamma_{\alpha}-\gamma_{\delta}= \pm 1$ and $\gamma_{\beta}-\gamma_{\delta}= \pm 1$ must hold simultaneously. From the latter it follows that $\gamma_{\delta}=1+\gamma_{\beta} \mp 1$, which gives for $\gamma_{\delta}$ the values $\gamma_{\beta}$ or $\gamma_{\beta}+2$, contrary to $\gamma_{\delta}=\gamma_{\beta} \mp 1$; hence $\gamma_{\alpha}$ is a dichotomous parameter, like $\sigma$. One of its values can be made to refer to polarization +1 , the other to -1 , the case of equal values being excluded by $\gamma_{\alpha}-\gamma_{\beta}= \pm 1$. They differ then in sign, so that $\gamma_{\beta}=-\gamma_{\alpha}=\gamma_{\alpha} \pm 1$, or $\gamma_{\alpha}= \pm 1 / 2$. This leads us to identify the values of the parameter $\gamma_{\alpha}$ with the eigenvalues $\pm 1 / 2$ of the spin projection in units of $\hbar$. Notice further that the vectors (17) become then eigenfunctions of the operator $-i \partial_{\varphi}$ with eigenvalues $\pm 1 / 2$, whence in this $\varphi$-representation $-\varphi$ being the internal rotation anglethe spin operator $\hat{S}_{z}$ becomes

$$
\hat{S}_{z}=-i \hbar \partial_{\varphi}
$$

in analogy with the orbital angular momentum operator $\hat{L}_{z}=-i \hbar \partial_{\phi}$. 


\section{Symmetry properties of the bipartite state vec- tor}

We recall that in nonrelativistic quantum mechanics the antisymmetry of the state vector for fermions is normally postulated, or borrowed from relativistic quantum field theory. As mentioned above, although there exist several proposed quantum-mechanical derivations of the spin-symmetry connection, the physical reasons for this connection remain unascertained (see Refs. [10] and [1] for a discussion).

In the following we will analyse the spin-symmetry connection by applying the tools of LSED to a stationary state of a two-electron system. This will prove to have the advantage of resorting to a more complete description of the state of the bipartite system, which involves the relevant field variables and the internal rotation angles in addition to the variables that represent the (quantum) states of the two particles according to the usual description. The symmetry properties of the bipartite state vector will therefore be determined by considering the exchange of the full set of variables.

A major outcome of LSED is that any stationary state of a system of two identical particles becomes described in terms of an entangled state vector of the form $([13$, 14] $)$

$$
|\psi\rangle_{12}^{A B}=\frac{1}{\sqrt{2}}\left(|A\rangle+\lambda_{A B}|B\rangle\right),
$$

with the entanglement parameter $\lambda_{A B}$ given by the product of the random field amplitudes, the subindices referring to particles 1 and 2,

$$
\lambda_{A B}=\left(e^{i \zeta_{\alpha \alpha^{\prime}}}\right)_{1}\left(e^{i \zeta_{\alpha^{\prime} \alpha}}\right)_{2} .
$$

In (19), state $|A\rangle$ is given by

$$
|A\rangle=\left|\alpha\left(\varphi_{1}\right)\right\rangle_{1}\left|\alpha^{\prime}\left(\varphi_{2}\right)\right\rangle_{2}=e^{-i \sigma \varphi_{1}}\left|\alpha_{0}, \sigma\right\rangle e^{-i \sigma^{\prime} \varphi_{2}}\left|\alpha_{0}^{\prime}, \sigma^{\prime}\right\rangle,
$$

and similarly for $|B\rangle$, with $\alpha, \alpha^{\prime}$ and $\sigma, \sigma^{\prime}$ interchanged; $A\left(\alpha, \alpha^{\prime}\right), B\left(\alpha^{\prime}, \alpha\right)$, with $\alpha^{\prime} \neq \alpha$, are different composite states having the same total energy $\mathcal{E}_{A}=\mathcal{E}_{\alpha}+\mathcal{E}_{\alpha^{\prime}}=\mathcal{E}_{B}$. In expressions such as (21), the first state vector refers always to particle 1 and the second one to particle 2. As is clear from (20), the entanglement results from the fact that identical particles couple through common relevant ZPF modes, which allows for the emergence of correlations between them.

Let us analyse the symmetry properties of the state vector $|\psi\rangle_{12}^{A B}$ under different exchange operations. The expression (19) lends itself to two such operations: one can either exchange states $A$ and $B$, or exchange particles 1 and 2 . In contrast to quantum mechanics, these operations are not equivalent because they act distinctly either on the particles (which are identical) or on the states (which are different, by construction). In particular, the exchange of particles involves the internal angular coordinate $\varphi$ (see below), a variable foreign to the usual quantum description. 
The first operation $(A, B) \rightarrow(B, A)$ leads to

$$
|\psi\rangle_{12}^{B A}=\frac{1}{\sqrt{2}}\left(|B\rangle+\lambda_{B A}|A\rangle\right) .
$$

According to (20), $\lambda_{B A}=\left(e^{i \zeta_{\alpha^{\prime} \alpha}}\right)_{1}\left(e^{i \zeta_{\alpha \alpha^{\prime}}}\right)_{2}=\lambda_{A B}^{*}=\lambda_{A B}^{-1}$, whence from (19) and (22),

$$
|\psi\rangle_{12}^{B A}=\lambda_{A B}^{-1}|\psi\rangle_{12}^{A B} \text {. }
$$

Since $|A\rangle$ and $|B\rangle$ represent two different states, in principle $|\psi\rangle_{12}^{B A}$ may be different from $|\psi\rangle_{12}^{A B}$.

Now we perform an exchange of particles $(1,2) \rightarrow(2,1)$. In contrast to the exchange of states, this one should have no effect on the composite state vector since the particles are identical, i.e.,

$$
|\psi\rangle_{21}^{A B}=|\psi\rangle_{12}^{A B} .
$$

For this operation we need to consider explicitly the dependence of $|\psi\rangle_{12}^{A B}$ on the intrinsic (internal) angular coordinate $\varphi$. As discussed in Ref. [12, one must take care that the rotations that take particle 1 to the azimuthal angle of particle 2 and vice versa, are both made in the same sense - say clockwise. When $\varphi_{2}>\varphi_{1}, \varphi_{1}$ transforms into $\varphi_{2}$ and $\varphi_{2}$ transforms into $2 \pi+\varphi_{1}$, so that one gets

$$
|\psi\rangle_{21}^{A B}=\frac{1}{\sqrt{2}}\left(e^{-2 \pi i \sigma^{\prime}}|B\rangle+\lambda_{B A} e^{-2 \pi i \sigma}|A\rangle\right)=-\frac{\lambda_{B A}}{\sqrt{2}}\left(\lambda_{A B}|B\rangle+|A\rangle\right),
$$

since $e^{-i 2 \pi \sigma}=e^{-i 2 \pi \sigma^{\prime}}=-1$ and $\lambda_{A B} \lambda_{B A}=1$. When $\varphi_{2}<\varphi_{1}, \varphi_{2}$ transforms into $\varphi_{1}, \varphi_{1}$ transforms into $2 \pi+\varphi_{2}$, and the exchange applied to $|\psi\rangle_{12}$ gives again Eq. (25). Therefore we have in both cases

$$
|\psi\rangle_{21}^{A B}=-\lambda_{B A}|\psi\rangle_{12}^{A B} .
$$

By comparing this result with Eq. (24) we obtain

$$
\lambda_{A B}=-1,
$$

which introduced into (19) or (23) leads to the well-known antisymmetric form of the state vector

$$
|\psi\rangle_{12}^{A B}=\frac{1}{\sqrt{2}}(|A\rangle-|B\rangle)=-|\psi\rangle_{12}^{B A},
$$

indicating that the permutation of fermion states $A \leftrightarrow B$ produces an overall change of sign, just as in quantum theory.

The above outcome has a further important physical implication: from (20) we note that $\lambda_{A B}=-1$ implies $\left(e^{i \zeta_{\alpha \alpha^{\prime}}}\right)_{1}=e^{\pi i}\left(e^{i \zeta_{\alpha \alpha^{\prime}}}\right)_{2}$, indicating that the coupling of particles 1 and 2 to the mode of frequency $\omega_{\alpha \alpha^{\prime}}$ occurs out of phase, with a phase difference of $\pi$. For particles with symmetric wave functions (i. e. 
with $\lambda_{A B}=1$ ), by contrast, the coupling occurs in phase, as seen from (20) 2 While an arbitrary number of (identical) particles can couple in phase to a single mode, no more than two particles can couple with a phase difference of $\pi$ to the same mode. This throws a new light on the Pauli exclusion principle.

\section{Final discussion}

The above analysis can be extended to a multielectron system, subject again to a common ZPF, thanks to the fact that the chain rule discussed in Sect. 3 remains in force for an arbitrary product of spin phases. To determine the resulting state of the system one must consider the various possible configurations $A, B, C, \ldots$ of stationary states corresponding to the same total energy $\mathcal{E}_{A}=\mathcal{E}_{B}=\mathcal{E}_{C}=\ldots$ Yet direct application of this procedure becomes rather cumbersome, as the degeneracy increases rapidly with the number of particles. A convenient approach is to consider first any pair of electrons of the system, say those in states $\alpha, \alpha^{\prime}$. By taking successively every possible pair, all relevant frequencies will be accounted for, and all the respective symmetries will thus be included. Since as a result no pair of electrons can be in the same (single-particle) state, the state of the entire system will be described by a totally antisymmetric, multiply entangled state vector built of different bipartite single-particle states that carry the factor $(-1)^{2 p \sigma}=(-1)^{p}$ in front of each term, where $p$ stands for the number of transpositions in the permutation needed to reach the corresponding exchanged state, starting from the initial state.

The calculations presented here confirm the physical picture of the spin of the electron as a helicoidal motion (a zitterbewegung) around the local mean trajectory, adding an effective structure to the originally pointlike particle. The operator $-i \hbar \partial_{\varphi}$ turns out to be the generator of internal rotations, according to Eq. (18). Further, for a bi- or multielectron system, consideration of the permanent presence of the background field resonantly connecting the particles serves to unveil the persistent mystery of the physics behind the spin-statistics association. The mediation of the ZPF turns out to have a definite role in fixing the statistics. Nevertheless, the above arguments appear so far to be insufficient to deal with the universe of bosonic particles, which in general are hadrons and subject also to nuclear interactions; other mechanisms most likely intervene in the definition of the total exchange symmetry properties of such compound systems.

Acknowledgments. The authors acknowledge helpful comments and suggestions from an anonymous referee. This work was supported in part by DGAPAUNAM, through project IN104816.

\footnotetext{
${ }^{2}$ Note that also in this latter case the assumption that the two-particle state vector does not change sign upon particle exchange, holds true.
} 


\section{References}

[1] E. Schrödinger 1930. Über die kräftefreie Bewegung in der relativistischen Quantenmechanik, Sitzungsber. Preuss. Akad. Wiss. Phys.-Math. Kl. 24, 418.

[2] H. J. Bhabha and H. C. Corben 1941. General Classical Theory of Spinning Particles in a Maxwell Field, Proc. Roy. Soc. A 178, 273.

[3] K. Huang 1952. On the Zitterbewegung of the Dirac Electron, Am. J. Phys. 20, 479 .

[4] P. A. M. Dirac 1958. The principles of quantum mechanics (Clarendon, Oxford), 4th edition, p. 262.

[5] L. de la Peña and A. Jáuregui 1982. The spin of the electron according to stochastic electrodynamics, Found. Phys. 12, 441.

[6] S. Sachidanandam 1983. A derivation of intrinsic spin one-half from random electrodynamics, Phys. Lett. A 97, 323.

[7] K. Muralidhar 2012. The Spin Bivector and Zeropoint Energy in Geometric Algebra, Adv. Studies Theor. Phys. 6, 675; see also by the same author: Classical Origin of Quantum Spin, Apeiron, 18 (2011), 146.

[8] D. Hestenes 1993. The kinematic origin of complex wave functions, in Physics and Probability. Essays in honor of Edwin T. Jaynes, W. T. Grandy Jr., and P. W. Milonni, eds. (Cambridge U. P., Cambridge).

[9] A. M. Cetto, L. de la Peña, and A. Valdés-Hernández 2014. Emergence of quantization: the spin of the electron, JPCS 504, 012007.

[10] I. G. Kaplan 2013. The Pauli Exclusion Principle. Can it be proved?, Found. Phys.43 1233-1251.

[11] I. G. Kaplan 2016. The Pauli Exclusion Principle: Origin, Verifications, and Applications (Wiley, 2017).

[12] A. Jabs 2010. Connecting spin and statistics in quantum mechanics, Found. Phys. 40, 776-792; see also arXiv:0810.2399v4 [quant-ph] 3 Feb 2014.

[13] L. de la Peña, A. M. Cetto, and A. Valdés-Hernández 2015. The emerging quantum. The physics behind quantum mechanics (Springer Verlag, Berlin).

[14] A. Valdés-Hernández, L. de la Peña and A.M. Cetto 2011. Bipartite Entanglement Induced by a Common Background (Zero-Point) Radiation Field, Found. Phys. 41, 843. A. M. Cetto and L. de la Peña 2016.

[15] A. M. Cetto and L. de la Peña 2016. Electron system correlated by the zeropoint field: physical explanation for the spin-statistics connection, JPCS 701, 012008. 
[16] I. I. Sobelman 1979. Atomic Spectra and Radiative Transitions (Springer Verlag, Berlin).

[17] L. Mandel and E. Wolf 1995. Optical Coherence and Quantum Optics (Cambridge U. P., Cambridge). 